\title{
NANOTECNOLOGIE PER CATTURARE LA LUCE
}

\author{
ANDREA LI BASSI (*) \\ Nota presentata dal m.e. Carlo E. Bottani \\ (Adunanza del 28 novembre 2013)
}

SunTO. - La trasformazione dell'energia solare in energia elettrica mediante l'impiego di celle solari è alla base della tecnologia solare fotovoltaica, che riveste un ruolo di primo piano nell'attuale ricerca di fonti energetiche alternative, sebbene non sia ancora competitiva con le sorgenti energetiche più tradizionali. Una delle sfide attuali è legata allo sviluppo di nuovi materiali e di nuovi tipi di celle solari che possano raggiungere efficienze di conversione energetica significative, con una contemporanea riduzione dei costi della produzione di energia; in questa sfida le nanotecnologie giocano un ruolo di primo piano. In questa nota vengono quindi presentati alcuni esempi di come le nanoscienze e le nanotecnologie possano fornire un contributo alla ricerca e allo sviluppo di materiali nanostrutturati per migliorare le proprietà delle celle solari di futura generazione. In particolare, dopo una parte introduttiva che presenta i vari tipi di celle solari e mostra perché le nanotecnologie possano fornire soluzioni promettenti in questo campo, si discuteranno due casi particolari: lo studio di nanostrutture gerarchiche di ossido di titanio come fotoanodi per celle solari a colorante di tipo innovativo, e lo sviluppo di nuovi ossidi nanostrutturati trasparenti e conduttivi come elettrodi multifunzionali per celle solari organiche o ibride.

$* * *$

ABSTRACT. - Solar photovoltaic technology is based on the direct conversion of solar radiation power into electrical power by means of solar cells. Photovoltaics can in principle play a major role in the search for alternative energy sources, even though it is still not competitive with respect to traditional sources, e.g. based on fossil fuels. One of the most important challenges of current scientific research in this field relates to the development of new solar cell materials or new cell architectures, possibly leading to an

(*) Dipartimento di Energia, Politecnico di Milano, Italy.

E-mail: andrea.libassi@polimi.it 
improvement of the cell power conversion efficiency or to the parallel reduction of the energy production cost; nanotechnology plays a leading role in this framework. In this paper selected examples will be presented, which show the possible contribution of nanoscience and nanotechnology to the study and optimization of nanostructured materials for better future generation solar cells. After an introductory part presenting the different types of solar cells and highlighting the role of nanotechnology research in this field, two specific cases will be presented and discussed: the study of hierarchical titanium oxide nanostructures as photoanodes for novel dye-sensitized solar cells and the development of nanostructured transparent conducting oxides for designing multifunctional electrodes for organic or hybrid photovoltaic cells.

\section{INTRODUZIONE}

Il soddisfacimento del fabbisogno energetico mondiale rappresenta una delle grandi sfide dell'umanità per il XXI secolo. Si pensi ad esempio che il solo consumo di energia elettrica nel mondo è oggi stimato essere dell'ordine dei 20000 TWh per anno. La ricerca di alternative ai combustibili fossili e i problemi di varia natura legati allo sfruttamento dell'energia nucleare (di sicurezza, sociali e politici, oppure tecnologici se si pensa alla tecnologia della fusione nucleare) hanno negli ultimi anni posto un forte accento sullo sviluppo delle cosiddette sorgenti energetiche alternative o rinnovabili. Tra queste un ruolo di rilievo, almeno a livello di potenzialità, è rivestito dalle tecnologie fotovoltaiche, ovvero le tecnologie che sfruttano la radiazione solare per la conversione in energia elettrica. Tale approccio è un esempio di conversione diretta dell'energia, ed in linea di principio può essere utilizzato sia per la produzione locale di energia (si pensi ai pannelli fotovoltaici di servizio a singole abitazioni, o per l'alimentazione di strumentazioni in luoghi isolati) sia per la produzione di energia in impianti fotovoltaici connessi alla rete elettrica. In aggiunta il fotovoltaico può essere pensato come soluzione energetica portabile (ad es. per caricare dispositivi elettronici, ecc.).

Tuttavia ad oggi il solare fotovoltaico risulta ancora marginalmente sfruttato (la stima della capacità totale di potenza fotovoltaica installata nel mondo, sebbene in continua crescita negli ultimi anni, nel 2013 si attestava intorno ai $140 \mathrm{GW}$ [1], contribuendo per una quota al di sotto dell' $1 \%$ alla produzione totale di potenza elettrica nel mondo) ed ancora non economicamente sostenibile. Altre problematiche che dovranno essere necessariamente affrontate affinché il fotovoltaico possa rivestire un ruolo di rilievo nella futura produzione energetica 
mondiale riguardano l'immagazzinamento dell'energia (legato allo sviluppo di batterie o accumulatori sempre più potenti ed efficienti) e la sua distribuzione, o ad esempio aspetti di tipo economico-politico legati alla sostituzione della produzione energetica basata sul petrolio.

Lungi dall'affrontare questi aspetti e dal fornire previsioni sul possibile ruolo e impatto del fotovoltaico nella società futura, questa nota vuole fornire un'idea ed alcuni esempi selezionati riguardanti il ruolo delle nanotecnologie nella ricerca scientifica volta allo sviluppo di nuovi materiali per il fotovoltaico o di celle innovative basate su concetti diversi da quelli su cui si basa il funzionamento della maggior parte delle celle solari oggi commercialmente disponibili (ovvero celle a silicio cristallino). Non verranno invece affrontati aspetti legati allo sviluppo tecnologico di pannelli o sistemi fotovoltaici, e altrettanto importanti quali la durabilità, la produzione su larga scala ed il conseguente impatto sui costi, l'integrazione in impianti o reti di distribuzione, ecc.

Infatti affinché la produzione di energia fotovoltaica diventi competitiva è necessario (anche se non sufficiente) che gli elementi attivi costitutivi dei pannelli fotovoltaici, ovvero le celle solari, diventino sempre più efficienti (dove per efficienza si intende l'efficienza di conversione da potenza di irraggiamento solare a potenza elettrica) e sempre meno costose. Perché questo sia possibile la ricerca si sta sempre più orientando allo sviluppo di nuovi materiali e nuove architetture di cella solare, e le nanotecnologie svolgono in questo un ruolo di rilievo, con la loro promessa di poter realizzare materiali ingegnerizzati con proprietà migliori rispetto ai materiali tradizionali (e quindi potenzialmente promettenti per il raggiungimento di elevate efficienze) o addirittura proprietà nuove (ad es. che permettano la realizzazione di celle solari flessibili implementabili in tessuti).

È utile qui definire le nanotecnologie come quelle tecnologie che sfruttano il confinamento quantico (cioè la dipendenza delle proprietà di un materiale dalla sua dimensione/dimensionalità quando si scende alla scala nanometrica, a causa delle leggi della meccanica quantistica) per la realizzazione di materiali o dispositivi con proprietà completamente nuove e modulabili rispetto ai materiali tradizionali. In un senso più lato poi le nanotecnologie riguardano in generale la realizzazione di materiali o sistemi (e dispositivi) con un controllo fine della loro struttura e delle loro proprietà alla scala nanometrica (o comunque sotto i $100 \mathrm{~nm}$ ), anche per ragioni di miniaturizzazione o di modulazione del rapporto superficie/volume. 
Uno dei paradigmi per la riduzione dei costi è poi l'abbinamento (o la sostituzione) dei materiali inorganici (semiconduttori), oggi utilizzati nelle celle solari, con materiali organici (nelle cosiddette celle solari organiche, o ibride), al fine di ridurre i costi. Va sottolineato fin da subito che questo tipo di ricerca è fortemente interdisciplinare, e necessita della convergenza di competenze negli ambiti della fisica, della chimica, della scienza dei materiali e dell'ingegneria.

Nei paragrafi successivi, dopo una veloce panoramica sulle diverse generazioni di celle solari e sui possibili benefici che un approccio nanotecnologico può apportare allo sviluppo di nuove celle, verrà descritto un tipo di cella solare elettrochimica a cui oggi la ricerca sta dedicando particolare attenzione (la cella a colorante o di Grätzel) e si discuteranno due esempi di come lo sviluppo di materiali nanostrutturati possa contribuire al miglioramento delle prestazioni di questo o altri tipi di celle.

\section{LE TRE GENERAZIONI DEL FOTOVOLTAICO}

Lo sviluppo della tecnologia fotovoltaica viene usualmente schematizzato nel succedersi di 3 generazioni di celle solari [2]:

$1^{a}$ generazione: celle solare a base di silicio (mono- o policristallino), rappresentano più dell' $80 \%$ delle celle solari attualmente in commercio. Le migliori efficienze di conversione energetica (power conversion efficiency) si attestano al di sotto del $30 \%$ sulla scala di laboratorio e al di sotto del $20 \%$ per un impianto fotovoltaico.

$2^{a}$ generazione: celle a film sottile, si tratta sempre di celle solari basate sull'impiego di semiconduttori inorganici, nelle quali però la riduzione dello spessore del materiale attivo alla scala micrometrica o submicrometrica può in prospettiva portare alla riduzione dei costi, anche se in genere questo è accompagnato da una riduzione dell'efficienza.

$3^{a}$ generazione: si tratta di un insieme di tipologie di concetti o architetture di cella, generalmente ancora al livello di ricerca di base o al più di prototipi, non ancora sul mercato, in cui le nanotecnologie vengono utilizzate per sfruttare le peculiari proprietà dei nanomateriali o diversi fenomeni fisici allo scopo di aumentare le efficienze oltre i limiti oggi possibili [3]; spesso viene fatta rientrare in questa categoria la ricerca riguardante le celle di tipo organico o ibrido, le cui efficienze sono invece attualmente inferiori a quelle delle celle a film sottile. 


\section{LA CELLA SOLARE, OVVERO UNA GIUNZIONE p-n ILLUMINATA}

È utile descrivere brevemente (ed in modo necessariamente semplice) su quali principi fisici si basa il funzionamento di una cella solare tradizionale (come quelle al silicio) che non è altro che una giunzione a semiconduttore p-n illuminata [2].

Un materiale semiconduttore è in grado di assorbire fotoni (quanti di energia elettromagnetica la cui energia è pari a $b v$, dove $b$ $\approx 6.63 \cdot 10^{-34} \mathrm{Js}$ è la costante di Planck e $v$ è la frequenza della radiazione) quando la loro energia è maggiore del gap energetico tra banda di valenza e banda di conduzione del materiale. Affiancando due semiconduttori (eventualmente lo stesso) drogati in modo diverso (ovvero con impurezze di tipo donore o accettore) si crea una giunzione p-n, ad alla giunzione si instaura un campo elettrico dovuto all'equilibrio che si crea a seguito della diffusione delle cariche maggioritare da un semiconduttore all'altro (negative, cioè elettroni nel caso di drogaggio $\mathrm{n}$, e positive, cioè buche o lacune nel caso di drogaggio p). La giunzione p-n è un diodo, che presenta una caratteristica corrente-tensione non lineare (rettificante). Quando tale giunzione viene illuminata vengono fotogenerati dei portatori di carica aggiuntivi (coppia elettrone-lacuna, in seguito all'assorbimento di un fotone e alla promozione di un elettrone dalla banda di valenza a quella di conduzione), i quali vengono poi separati e trasportati in direzioni diverse dal campo elettrico della giunzione (Fig. 1), dando origine al flusso di una corrente elettrica che può essere sfruttata per alimentare un carico esterno (si veda in Fig. 2 la tipica caratteristica corrente-tensione di una cella fotovoltaica).

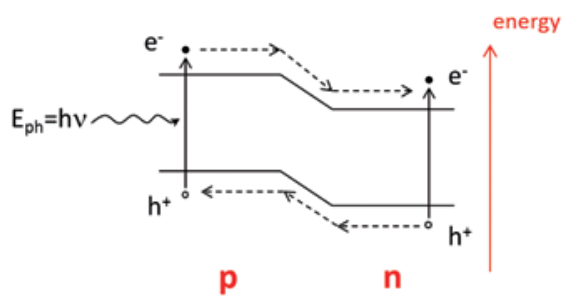

Fig. 1 - Schema delle bande di energia in una giunzione p-n. 


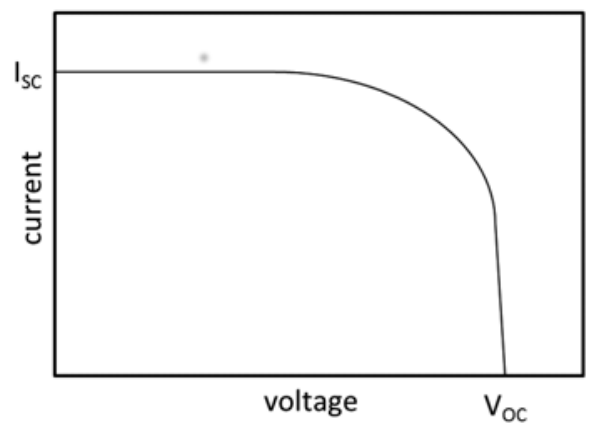

Fig. 2 - Tipica caratteristica tensione-corrente di una cella solare.

\section{Il POSSIBILE RUOLO DELLE NANOTECNOLOGIE NEL FOTOVOLTAICO DI TERZA GENERAZIONE}

Risulta intuitivo che l'efficienza di assorbimento del materiale attivo di una cella è tanto maggiore quanto più piccolo è il gap, dal momento che anche fotoni di bassa energia saranno assorbiti, e questo influenza direttamente il numero di portatori fotogenerati e quindi la massima corrente ottenibile (ISC, corrente a circuito chiuso) mentre la massima tensione che si può ottenere ai capi della cella (VOC, tensione di circuito aperto) è limitata dal gap energetico, che fornisce il massimo potenziale chimico della coppia elettrone-lacuna fotogenerati $\left(E_{\mathrm{g}} / e\right.$, con $E_{\mathrm{g}}$ gap di energia ed $e$ carica dell'elettrone). Pertanto c'è un tradeoff tra i due aspetti ed un'analisi quantitativa (dovuta orginariamente a Shockley e Queisser) mostra che il gap 'ottimale' è ad un valore intermedio di circa $1.4 \mathrm{eV}$, per il quale la massima efficienza teorica è di poco superiore al $30 \%$ [2], considerando uno spettro di radiazione distribuito su varie frequenze come quello solare (principalmente nel visibile e nell'IR). Il gap del Si è pari a $1.1 \mathrm{eV}$.

Al fine di superare questo limite intrinseco delle celle basate su una giunzione $\mathrm{p}-\mathrm{n}$ è necessario poter estrarre più lavoro (cioè energia) per ogni singolo fotone incidente sulla cella, ad es. aumentandone la probabilità di assorbimento; o facendo in modo che tutta l'energia del fotone sia convertita in potenziale chimico (e quindi elettrico), ad es. minimizzando le perdite di energia dei portatori di carica sotto forma di calore (dovute agli urti elettrone-fonone); ciò porterebbe ad un aumento della tensione di cella, ed in questo caso si parla di hot carrier cells. Un'altra 
strada è quella di fare in modo che ad un singolo fotone corrisponda la generazione di più coppie elettrone-lacuna (multiple carrier generation), il che può portare ad un aumento della corrente di cella.

Esistono oggi varie idee o concetti, basati sull'uso di nanostrutture o sulle particolari proprietà di strutture quantiche, su come implementare queste possibili soluzioni in dispositivi reali [3], anche se va detto che spesso queste idee sono ad uno stadio molto prematuro di sviluppo sperimentale ed in molti casi resta ancora da dimostrare l'effettiva possibilità di sfruttare questi fenomeni in una vera e propria cella solare.

Non è questo il luogo dove approfondire queste tematiche. Basti qui citare l'interesse nel possibile uso di punti quantici (quantum dots, QD, ovvero nanoparticelle di semiconduttori che presentano confinamento quantico) in celle solari di futura generazione, grazie alla possibilità di modulare il gap energetico di un materiale controllandone la dimensione alla scala nanometrica, e di realizzare così celle a multigiunzione (o tandem) in grado di sfruttare in modo ottimale lo spettro della radiazione solare. Inoltre i fenomeni di multiple carrier generation e soppressione dell'interazione elettrone-fonone sembrano essere favoriti in strutture che presentano confinamento quantico, come i QD.

Nel seguito verranno invece discussi due esempi relativi allo sviluppo di ossidi nanostrutturati di possibile interesse come elettrodi (o fotoelettrodi) in celle solari di tipo elettrochimico od organico.

\section{LA CELLA SOLARE A COLORANTE, O DI GRÄTZEL}

Accanto all'ambito degli studi riguardanti celle organiche o ibride che utilizzino materiali quali molecole e polimeri, un posto particolare meritano le cosiddette celle solari a colorante (dye-sensitized solar cells, DSSC, o celle di Grätzel dal nome del loro inventore e principale studioso).

Nel 1991 Gräztel e O’Regan [4] hanno proposto un tipo di cella solare elettrochimica nella quale l'anodo è realizzato con un ossido nanoporoso di elevata area superficiale (il fotoanodo, un film di nanoparticelle di biossido di titanio dello spessore di qualche decina di micron), la cui superficie è rivestita da un monostrato di molecole coloranti $(d y e)$ in grado di assorbire la luce. In particolare l'assorbimento di un fotone porta la molecola in uno stato eccitato, con conseguente iniezione di un elettrone nella banda di conduzione del fotoanodo; l'elettrone è poi trasportato all'elettrodo trasparente su cui il fotoanodo 
poroso è deposto (elettrodo a base di un ossido trasparente conduttivo, ovvero un transparent conducting oxide, TCO; tipicamente si tratta di FTO, ovvero fluorine-doped tin oxide, $\mathrm{F}: \mathrm{SnO}_{2}$ ); poi attraverso il carico esterno, dove compie lavoro elettrico, fino al controelettrodo. Il circuito della cella è chiuso da un elettrolita (normalmente liquido) contenente una coppia redox in grado di trasportare l'elettrone dal catodo fino alla molecola (o di trasportare la buca dalla molecola ionizzata al catodo), permettendone così la riduzione e chiudendo il ciclo (vedi Fig. 3). Si tratta di un tipo di cella solare appartenente alla cosiddetta classe delle celle eccitoniche, e nella quale l'assorbimento dei fotoni ed il trasporto della carica avvengono in materiali diversi. La corrente di circuito chiuso della cella dipende dal numero di molecole ancorate alla superficie del fotoanodo ed in grado di assorbire fotoni; la tensione di circuito aperto dipende invece dalla differenza tra il livello di Fermi nel fotoanodo ed il potenziale di riduzione (redox) dell'elettrolita, che misura la sua capacità di acquisire elettroni. Recenti sviluppi di ricerca hanno portato l'attenzione a varianti delle DSSC in cui l'elettrolita è solido (un polimero trasportatore di buche), con vantaggi in termini di stabilità e durabilità della cella (DSSC a stato solido) [5].

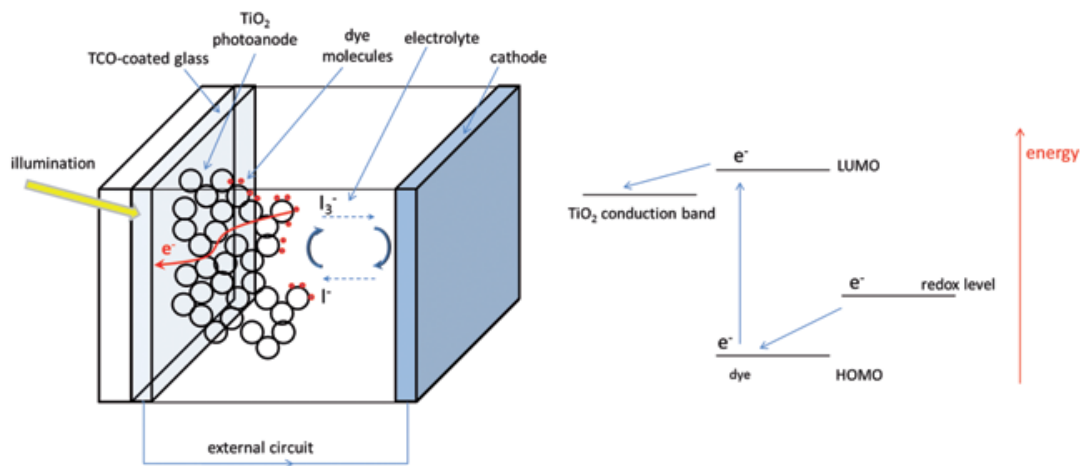

Fig. 3 - A sinistra, schema dell'architettura di una cella solare a colorante (DSSC); a destra, diagramma dei livelli energetici coinvolti nel funzionamento della cella.

Nel seguito di questa nota ci focalizzeremo sulle proprietà del fotoanodo di $\mathrm{TiO}_{2}$ e sull'elettrodo trasparente a base di TCO e discuteremo in che modo un controllo della struttura del materiale alla scala nanometrica possa essere sfruttato per migliorare le prestazioni di questi elementi della cella. 
Vale la pena notare, a questo punto, che le DSSC sono, fra le celle cosiddette di terza generazione, quelle probabilmente con uno sviluppo tecnologico più avanzato e più vicine ad una possibile applicazione commerciale diffusa (sono già presenti sul mercato in misura limitata). La loro efficienza è, nell'ambito delle celle eccitoniche, elevata (record di laboratorio $>12 \%$, e ancora maggiore nel caso di DSSC a stato solido con perovskiti quale materiale assorbitore al posto delle molecole di colorante [6]); si stima che i costi di produzione possano essere contenuti e i materiali utilizzati sono generalmente abbondanti e sicuri. La possibilità di costruire le celle su substrati plastici e di modularne il colore attraverso la scelta del colorante le rende ad esempio appetibili per un loro utilizzo in applicazioni portabili, o per integrazione in elementi architettonici di edifici ad alta tecnologia: building-integrated photovoltaics, BIPV.

\subsection{Fotoanodi a base di nanostrutture gerarchiche di $\mathrm{TiO}_{2}$}

Una delle caratteristiche principali delle DSSC è la struttura nanoporosa del fotoanodo, che permette di raggiungere elevate aree superficiali, dell'ordine delle centinaia $\mathrm{di}^{2} / \mathrm{g}$, o delle centinaia o migliaia di $\mathrm{cm}^{2}$ per $\mathrm{cm}^{2}$ di superficie geometrica della cella (il cosiddetto roughness factor). Questo significa che un numero molto elevato di molecole possa essere depositato sull'unità di area geometrica della cella, permettendo un efficace assorbimento della luce e pertanto un'elevata corrente elettrica (valori tipici sono dell'ordine di decine di $\mathrm{mA} / \mathrm{cm}^{2}$, mentre la tensione di circuito aperto per le DSSC è dell'ordine di $0.7 \mathrm{~V}$, e determinato dalla differenza tra l'energia di Fermi del fotoanodo di $\mathrm{TiO}_{2}$ e il livello energetico redox dell'elettrolita).

Il trasporto di elettroni attraverso la rete di nanoparticelle di $\mathrm{TiO}_{2}$ potrebbe essere ottimizzato utilizzando nanostrutture monodimensionali (1D), con trasporto direzionale dal punto di iniezione da parte della molecola fino all'elettrodo trasparente, ma in genere nanofili e nanotubi di $\mathrm{TiO}_{2}$ (o altri ossidi) hanno un'area superficiale limitata. Una soluzione per combinare elevata area superficiale e la direzionalità del trasporto di carica è l'utilizzo di strutture quasi-1D, come le nanostrutture gerarchiche, ad albero, mostrate in Fig. 4 e ottenute mediante deposizione da laser pulsato dal nostro gruppo di ricerca.

La deposizione da laser pulsato (in inglese Pulsed Laser 
Deposition, PLD) consiste nella vaporizzazione (ablazione) di un bersaglio solido da parte di impulsi laser (solitamente nell'UV) di elevata energia (centinaia di $\mathrm{mJ}$ ) e durata breve (tipicamente nell'ordine dei nanosecondi). Se la vaporizzazione avviene in presenza di un'atmosfera inerte o reattiva, può avvenire nucleazione di nanoparticelle nella cosiddetta piuma di ablazione, con conseguente loro rallentamento dovuto alla diffusione nel gas [7]. Questo permette la deposizione di nanoparticelle con ridotta energia cinetica, che può così essere sfruttata per la crescita di film nanoporosi e, in opportune condizioni, di film con struttura gerarchica come quelli in Fig. 4, la cui struttura cristallina può essere poi eventualmente controllata/modificata mediante trattamenti termici post-deposizione [8].
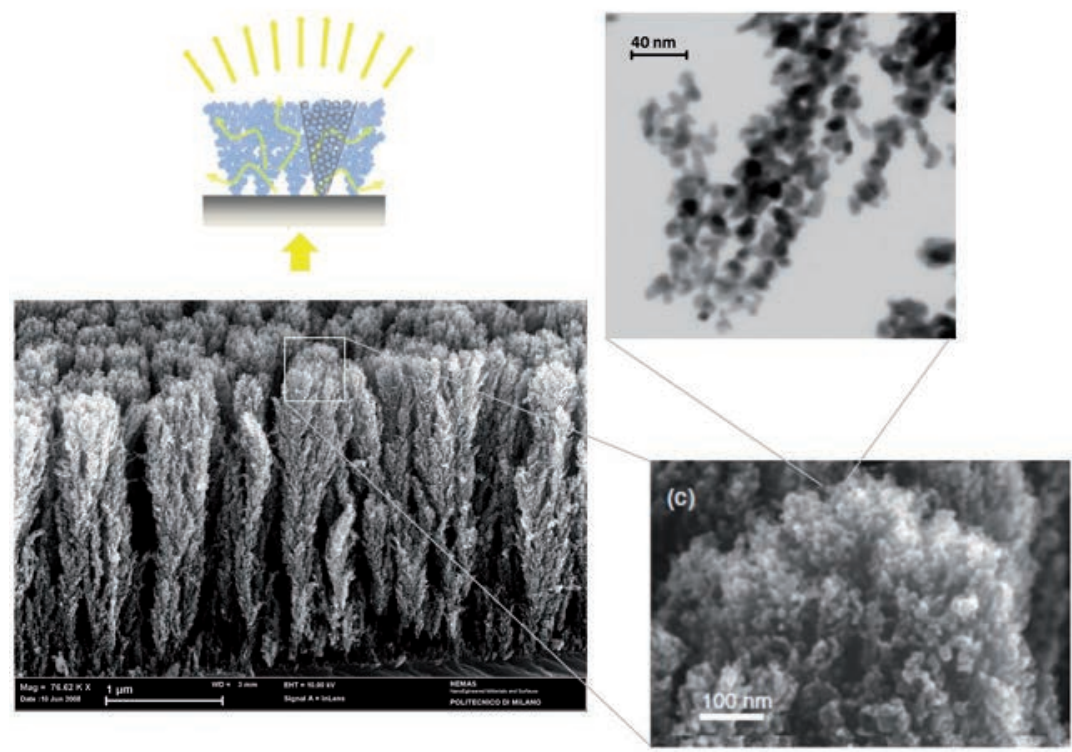

Fig. 4 - Immagini al microscopio elettronico (SEM) a diversi ingrandimenti di nanostrutture gerarchiche (ad 'albero') di ossido di titanio ottenute mediante ablazione laser; in alto a sinistra, rappresentazione schematica dell'effetto di diffusione di luce dovuti agli 'alberi' di $\mathrm{TiO}_{2}$.

Una struttura di tipo gerarchico (e quindi multiscala, ovvero con una morfologia che presenti organizzazione e lunghezze caratteristiche a scale diverse) può inoltre presentare altri vantaggi per l'applicazione 
di interesse [9]. Infatti, se da una parte la struttura nanometrica e nanoporosa (dovuta al fatto che il materiale è costituito da mattoni fondamentali rappresentati dalle nanoparticelle) garantisce un'elevata area superficiale interna (superiore a quella di strutture puramente $1 \mathrm{D}$ come quelle sopra citate), dall'altra l'organizzazione gerarchica garantisce sia una orientazione preferenziale verticale (che come detto può essere benefica per il trasporto di carica verso l'elettrodo), sia la presenza di metastrutture (gli 'alberi') con dimensioni caratteristiche dell'ordine delle centinaia di nm, ovvero confrontabili con la lunghezza d'onda della radiazione nella regione visibile dello spettro solare. Questo potenzialmente garantisce un'efficace ed elevata diffusione della luce (in inglese scattering) in direzioni diverse da quella della radiazione incidente, aumentando così il percorso della luce nella cella e favorendone l'interazione con le molecole fotoassorbenti sulla superficie delle particelle: si parla in questi casi di light trapping (intrappolamento) o barvesting (raccolta). Inoltre la presenza di canali mesoporosi (decine di $\mathrm{nm}$ ) favorisce l'infiltrazione di un eventuale elettrolita polimerico, che spesso è un problema nello sviluppo di DSSC a stato solido.

Nanostrutture gerarchiche di ossido di titanio sono state testate come fotoanodi in DSSC ad elettrolita liquido [10] (mostrando un aumento del tempo di vita medio degli elettroni iniettati nell'ossido, e quindi delle proprietà di trasporto elettrico), ed in DSSC a stato solido (polimeriche) [11], per le quali si è dimostrato che l'effetto di diffusione della luce porta ad un aumento dell'assorbimento da parte delle molecole di dye e di conseguenza ad un aumento dell'efficienza di conversione energetica (dal $2.3 \%$ al $4 \%$, in prototipi alla scala di laboratorio) rispetto all'utilizzo di fotoanodi di tipo tradizionale ottenuti da paste di nanoparticelle di $\mathrm{TiO}_{2}$.

\section{OSSIDI TRASPARENTI E CONDUTTIVI NANOSTRUTTURATI}

Abbiamo citato l'elettrodo trasparente nelle DSSC. Un elettrodo trasparente è necessario in qualsiasi tipo di cella solare a film sottile, poiché una delle due 'facce' della cella deve essere in grado di permettere il 'passaggio' della radiazione fino al materiale attivo della cella e allo stesso tempo permettere il trasporto di carica elettrica verso il circuito esterno. Non è ovviamente l'unica applicazione dei TCO, si pensi ad esempio ai touch screen o all'elettronica trasparente. 
Solitamente la combinazione di trasparenza ottica e buona conducibilità elettrica non è tipica dei materiali 'tradizionali' (come i metalli), e può essere ottenuta mediante il drogaggio pesante di un ossido con gap ottico molto ampio $(>3 \mathrm{eV})$; i TCO sono materiali che in alcuni casi hanno ormai raggiunto uno sviluppo tecnologico maturo.

È però oggi interessante sviluppare nuovi TCO che a queste caratteristiche affianchino nuove funzionalità, come la compatibilità con substrati plastici od organici per applicazioni flessibili (il che richiede basse o moderate temperature di sintesi); la capacità non solo di trasmettere, ma di intrappolare o dirigere la luce all'interno della cella; la possibilità di realizzare TCO con elevata area superficiale per lo sviluppo di interfacce complesse ad esempio con materiali organici.

Mediante ablazione laser è possibile realizzare TCO, ad esempio di ossido di zinco drogato con alluminio $(\mathrm{Al}: \mathrm{ZnO}$, con un contenuto di $\mathrm{Al} \sim 3 \%$ at.) con proprietà stato dell'arte (trasparenza dell'ordine del $90 \%$ e conducibilità elettrica dell'ordine di $10^{-4} \Omega \mathrm{cm}$ ), con una sintesi a temperatura ambiente, e quindi compatibile con substrati plastici e quindi celle solari organiche [12].

In Fig. 5 è mostrato invece un esempio di struttura multifunzionale di ossido di zinco drogato con alluminio $(\mathrm{Al}: \mathrm{ZnO})$ realizzata sempre mediante ablazione laser, e ottenuta modulando la pressione dell'atmosfera di deposizione in modo che lo strato sottostante (deposto ad alta p, $100 \mathrm{~Pa}$ di $\mathrm{O}_{2}$ ) sia composto da nanoparticelle e quindi nanoporoso/gerarchico, in grado di diffondere la luce incidente sul film (generalmente da un sottostante substrato di vetro) in tutte le direzioni, mentre lo strato superiore compatto (deposto a bassa p, $2 \mathrm{~Pa} \mathrm{di}_{2}$ ) è caratterizzato da elevata conducibilità elettrica. Tale struttura a gradiente di morfologia risulta avere una trasparenza $>80 \%$, una conducibilità efficace dell'ordine dei $10^{-3} \Omega \mathrm{cm}$, ed in aggiunta una capacità di diffondere la luce (misurata mediante un parametro detto baze) superiore al $40 \%$. Ovviamente questi valori possono essere ottimizzati, ma mostrano la possibilità di combinare diverse proprietà funzionali in un unico materiale con struttura ingegnerizzata alla nanoscala.

Utilizzando elettrodi con questa struttura è stato possibile mostrare che l'assorbimento della radiazione nel visibile di un polimero fotoattivo (PCPDTBT) depositato sulla superficie del film aumenta significativamente (in pratica raddoppia), rispetto alla situazione in cui lo stesso polimero è deposto su un elettrodo trasparente tradizionale ('compatto') [13]. Questo apre la strada al potenziale aumento dell'efficienza di 
celle solari a film sottile, ad esempio di tipo organico (in particolare le cosiddette bulk beterojunctions [14]).

Un'altra possibilità è quella di realizzare una nanostruttura gerarchica/nanoporosa in grado di diffondere la luce nella cella, e di agire invece sulla composizione e sulla chimica dei difetti del materiale per aumentarne ed ottimizzarne la conducibilità elettrica.

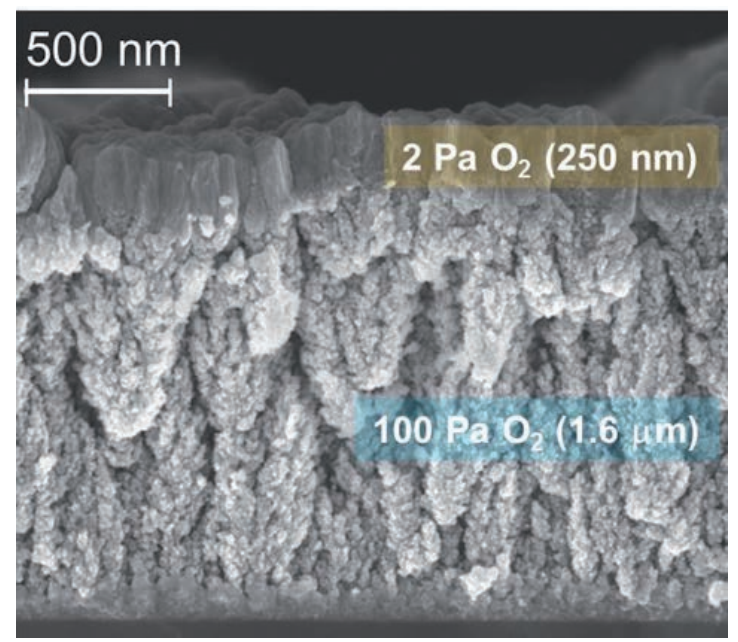

Fig. 5 - Immagine al microscopio elettronico (SEM) del film di Al: $\mathrm{ZnO}$

(TCO 'multifunzionale') con gradiente di morfologia ('compatto' su nanoporoso).

\section{CONCLUSIONI}

Questa nota ha mostrato solo due esempi, tratti dalla nostra esperienza di ricerca, di come il controllo della struttura dei materiali alla scala atomica e nanometrica possa essere utilizzato per migliorare le prestazioni di dispositivi con architetture innovative per la conversione fotovoltaica, e rappresenta solo un assaggio della potenzialità (in linea di principio anche più significative e radicali di quelle qui discusse) dell'applicazione delle nanotecnologie in questo campo. Ovviamente molto resta da capire e molto è il lavoro che resta da fare prima che questi materiali e questi dispositivi possano essere effettivamente utilizzati e commercializzati (e per capire se questo sia possibile).

Vale la pena, a conclusione, notare due cose. La ricerca in questo 
campo (nanoscienze, nanomateriali, nanotecnologie per il solare fotovoltaico) può portare allo sviluppo di materiali innovativi, di interesse anche per altri campi di applicazione (si pensi alla fotocatalisi, per la quale le proprietà di interazione dei materiali con la luce sono ovviamente fondamentali). Inoltre spesso tale tipo di ricerca porta a significative ricadute in termini di ricerca di base, ad esempio legate alla comprensione di nuovi fenomeni fisici alla scala nanometrica o della relazione tra struttura e proprietà funzionali in materiali di nuova concezione.

\section{RINGRAZIAMENTI}

L'autore ringrazia tutti i ricercatori del Laboratorio Materiali Micro e Nanostrutturati del Dipartimento di Energia, Politecnico di Milano, e tutti gli altri collaboratori che hanno contribuito ai risultati presentati in questa nota (paragrafi 5.1 e 6), in particolare i coautori delle pubblicazioni n. 7, 8, 10, 11, 12, 13 .

\section{BIBLIOGRAFIA}

[1] Renewables 2014 Global Status Report, REN21 Secretariat, Paris, 2014 [see www.ren21.net].

[2] J. Nelson, The Physics of Solar Cells, Imperial College Press, London, 2003.

[3] G. Conibeer, Third-generation photovoltaics, Materials Today, 2007: 10: 42-50.

[4] B. O'Regan and M. Grätzel, A low-cost, high efficiency solar cell based on dyesensitized colloidal $\mathrm{TiO}_{2}$ films, Nature, 1991: 353: 737-740.

[5] H.J. Snaith and L. Schmidt-Mende, Advances in Liquid-Electrolyte and SolidState Dye-Sensitized Solar Cells, Advanced Materials, 2007: 19: 3187-3200.

[6] H.J. Snaith, Perovskites: The Emergence of a New Era for Low-Cost, HighEfficiency Solar Cells, Journal of Physical Chemistry Letters 2013: 4: 3623-3630.

[7] C.S. Casari and A. Li Bassi, Pulsed Laser Deposition of Nanostructured Oxides: from Clusters to Functional Films, in Advances in Laser and Optics Research, ed. W.T. Arkin, vol.7, 65-100, Nova Science Publishers Inc, 2012.

[8] F. Di Fonzo, C.S. Casari, V. Russo, M.F. Brunella, A. Li Bassi, C.E. Bottani, Hierarchically organized nanostructured $\mathrm{TiO}_{2}$ for photocatalysis applications, Nanotechnology, 2009: 20: 015604/1-7.

[9] Q. Zhang and G. Cao, Hierarchically structured photoelectrodes for dye-sensitized solar cells, Journal of Materials Chemistry, 2011: 21: 6769-6774.

[10] F. Sauvage, F. Di Fonzo, A. Li Bassi, C.S. Casari, V. Russo, G. Divitini, C. Ducati, C.E. Bottani, P. Comte, M. Grätzel, Hierarchical TiO2 Photoanode for DyeSensitized Solar Cells, Nano Letters 2010: 10: 2562-2567. 
[11] L. Passoni, F. Ghods, P. Docampo, A. Abrusci, J. Martì-Rujas, M. Ghidelli, G. Divitini, C. Ducati, M. Binda, S. Guarnera, A. Li Bassi, C.S. Casari, H.J. Snaith, A. Petrozza, F. Di Fonzo, Hyperbranched Quasi-1D Nanostructures for SolidState Dye-Sensitized Solar Cells, ACS Nano, 2013: 11: 10023-10031.

[12] P. Gondoni, M. Ghidelli, F. Di Fonzo, M. Carminati, V. Russo, A. Li Bassi, C.S. Casari, Structure-dependent optical and electrical transport properties of nanostructured Al-doped ZnO, Nanotechnology, 2012: 23: 365706/1-8.

[13] Gondoni, P. Mazzolini, V. Russo, A. Petrozza, A.K. Srivastava, A. Li Bassi, C.S. Casari, Enhancing light harvesting by hierarchical functionally graded transparent conducting Al-doped $\mathrm{ZnO}$ nano- and mesoarchitectures, Solar Energy Materials E Solar Cells, 2014: 128: 248-253.

[14] A.C. Mayer, S.R. Scully, B.E. Hardin, M.W. Rowell, M.D. McGehee, Polymerbased solar Cells, Materials Today, 2007: 11: 28-33. 